\title{
Unsteady Flow of a Dusty Visco-Elastic Fluid through an Inclined Channel
}

\author{
Geetanjali Alle ${ }^{1}$, Aashis S. Roy ${ }^{2}$, Sangshetty Kalyane ${ }^{1}$, Ravi M. Sonth ${ }^{3}$ \\ ${ }^{1}$ Department of Physics, Singhania University, Rajasthan, India \\ ${ }^{2}$ Department of Materials Science, Gulbarga University, Gulbarga, India \\ ${ }^{3}$ Department of Mathematics, K.C.T. Engineering College, Gulbarga, India \\ E-mail: principalkct@rediffmail.com \\ Received March 1, 2011; revised April 26, 2011; accepted May 10, 2011
}

\begin{abstract}
The present discussion deals with the study of an unsteady flow of a dusty fluid through an inclined channel under the influence of pulsatile pressure gradient along with the effect of a uniform magnetic field. The analytical solutions of the problem are obtained using variable separable and Fourier transform techniques. The graphs drawn for the velocity fields of both fluid and dust phase under the effect of Reynolds number. The velocity profiles for the liquid and the dust particles decreases at different values of time $t$ increases. As the visco-elastic parameter $\lambda$ increases the velocity of the liquid and the dust particles deceases. When relaxation time parameter $\sigma$ increases, the velocity of the liquid and dust particles decreases.
\end{abstract}

Keywords: Dusty Fluid, Pulsatile Pressure Gradient, Velocities of Dust and Fluid Phase, Inclined Channel, Reynolds Number

\section{Introduction}

In recent years many authors have studied the flow of immiscible viscous electrically conducting fluids and their different transport phenomena. These fluid also known as non-Newtonian fluids are molten plastics. Plups, emulsion etc., and large variety of industrial product having visco-elastic behavior in their motion. Such fluids are often embedded with spherical nonconducting dust particles in the form of impurities. This fluid also called dusty Rivlin-Ericksen second order fluid. The influence of dust particles on visco-elastic fluid flow has its importance in many applications such as extrusion of plastic in the manufacture of rayon and Nylon, purification of crude oil, pulp oil, pulp, paper industry, textile industry and in different geophysical cases etc. In these cases stratification effect is often observed which are under the action of geomagnetic field.

Saffman et al., (1962) studied the stability of a laminar flow of dusty gas with uniform distribution of dust particles. Michel (1965) considered the Kelvin-Helmholtz instability of the dusty gas. Michael and Miller (1965) discussed the motion of the dusty gas enclosed in the same infinite space above a rigid plane boundary. We have studied the unsteady dusty visco-elastic liquid in a channel bounded by two parallel plates. The change in velocity profiles for dust and liquid particles has been depicted graphically.

\section{Theory}

\section{Formulation and Solution of the Problem}

The $\mathrm{X}$-axis is taken along the plate and the $\mathrm{Y}$-axis normal to it. The basic equations of hydro magnetic flow are

$$
\begin{aligned}
& \frac{\partial u_{1}^{\prime}}{\partial t^{\prime}}+\left(u_{1}^{\prime} \cdot \nabla\right) u_{1}^{\prime}=-\frac{1}{\rho} \nabla \cdot P^{\prime}+\left(\gamma^{\prime}+\beta \nabla\right) \nabla^{2} u_{1}^{\prime} \\
& +\frac{k N_{0}}{\rho^{\prime}}\left(u_{2}^{\prime}-u_{1}^{\prime}\right) \\
& \frac{\partial u_{2}^{\prime}}{\partial t^{\prime}}+\left(u_{2}^{\prime} \cdot \nabla\right) u_{2}^{\prime}=\frac{k}{m}\left(u_{1}^{\prime}-u_{2}^{\prime}\right) \\
& \operatorname{div} u_{1}^{\prime}=0 \\
& \operatorname{div} u_{2}^{\prime}=0
\end{aligned}
$$

where the $u_{1}^{\prime}, u_{2}^{\prime}$ denotes the velocity vector of fluid and dust particles respectively: $p^{\prime}$ the pressure: $\rho^{\prime}$ the density of the fluid: $\gamma^{\prime}$ kinematic coefficient of viscosity: $t^{\prime}$ the time: $\mathrm{m}$, the mass of the dust particles: $N_{0}$, the number density of dust particles: $K$, the stokes 
resistance coefficient which for spherical particles of radius a is $6 \pi \mu^{\prime}$ a: $\mu$, the coefficient of viscosity of fluid particles.

In the present analysis, the following important assumptions are made:

1) The dust particles are spherical in shape are uniformly distributed.

2) Chemical reaction, mass transfer and radiation between the particles and fluid are not considered.

3) The temperature is uniform within a particle.

4) Interaction between particles themselves is not considered.

5) The flow is fully developed.

6) The buoyancy force is neglected.

7) The number density of the dust particles is constant throughout the motion.

8) The displacement current is zero, since the flow velocity is small relative to the speed of light.

9) The Hall effects are negligible.

10) The fluid is electrically neutral, i.e., no surplus electrical charge distribution is present in the fluid.

11) Only the electromagnetic body forces are present.

12) Fluid properties are invariable.

13) Viscous dissipation is neglected.

Maxwell's equations, together with Ohm's law and the law of electromagnetic conservation, are written in the case of zero-displacement and hall currents as:

$$
\begin{aligned}
& \nabla \times \boldsymbol{B}=\boldsymbol{J} \\
& \nabla \times \boldsymbol{E}=\frac{-\partial \boldsymbol{B}}{\partial t} \\
& \boldsymbol{J}=\sigma_{1}(E+\boldsymbol{V} \times \boldsymbol{B}) \\
& \nabla \cdot \boldsymbol{B}=0 \\
& \nabla \cdot \boldsymbol{E}=0
\end{aligned}
$$

The usual Prandtl boundary layer assumptions along with assumptions (5)-(9) leads to the following reduction of the previous equations:

$$
\begin{gathered}
\frac{\partial u_{1}^{\prime}}{\partial t^{\prime}}=-\frac{1}{\rho} \frac{\partial P^{\prime}}{\partial x^{\prime}}+\left(\gamma^{\prime}+\beta \frac{\partial}{\partial t^{\prime}}\right) \frac{\partial^{2} u_{1}^{\prime}}{\partial y^{2}}+\frac{K N_{0}}{\rho}\left(u_{2}^{\prime}-u_{1}^{\prime}\right) \\
\frac{\partial u_{2}^{\prime}}{\partial t^{\prime}}=K\left(u_{1}^{\prime}-u_{2}^{\prime}\right)
\end{gathered}
$$

which are to be solved subject to the boundary conditions

$$
\begin{aligned}
& t^{\prime}=0, \quad u_{1}^{\prime}=u_{2}^{\prime}=0 \\
& t^{\prime}>0, \quad-\frac{1}{\rho} \frac{\partial P^{\prime}}{\partial x^{\prime}}=C \text { (constant) } \\
& y^{\prime}= \pm h, \quad u_{1}^{\prime}=0, \quad u_{2}^{\prime}=0
\end{aligned}
$$

Changing it into non dimensional form by putting

$$
y=\frac{y^{\prime}}{h}, x=\frac{x^{\prime}}{h}, t=\frac{\gamma^{\prime} t^{\prime}}{h^{2}}, u=\frac{u_{1}^{\prime} h}{\gamma^{\prime}}, v=\frac{u_{2}^{\prime}}{\gamma^{\prime}}, p=\frac{p^{\prime} h^{2}}{\rho^{\prime} \gamma^{\prime 2}}
$$

We have

$$
\begin{gathered}
\frac{\partial u}{\partial t}=-\frac{\partial p}{\partial x}+\left(1-\lambda \frac{\partial}{\partial t}\right) \frac{\partial^{2} u}{\partial y^{2}}+\frac{l}{\sigma}(v-u) \\
\sigma \frac{\partial v}{\partial t}=u-v
\end{gathered}
$$

where

$$
\sigma=\frac{m \gamma^{\prime}}{K h^{2}} \quad \text { Relaxation time parameter, } \quad l=\frac{m N_{0}}{\rho}
$$

Mass Concentration $\lambda=\frac{-\beta}{h^{2}}$ Visco-elastic parameter

The boundary conditions are

$$
\begin{array}{ll}
t=0: & u=0, \quad v=0 \\
t>0: & u=0, \text { at } y=-1 \\
& u=0, \text { at } y=1
\end{array}
$$

take $-\frac{\partial p}{\partial x}=C$ (constant) for $t>0$.

Then the equation (2.2.13) becomes

$$
\frac{\partial u}{\partial t}=C+\left(1-\lambda \frac{\partial}{\partial t}\right) \frac{\partial^{2} u}{\partial y^{2}}+\frac{l}{\sigma}(v-u)
$$

Appling the Laplace Transform, we have from (14) and (16)

$$
\begin{gathered}
S \bar{u}=\frac{C}{S}+(1-\lambda S) \frac{\partial^{2} \bar{u}}{\partial y^{2}}+\frac{l}{\sigma}(\bar{v}-\bar{u}) \\
\sigma S \bar{v}=\bar{u}-\bar{v}
\end{gathered}
$$

where

$$
\bar{u}=\int_{0}^{\infty} u \mathrm{e}^{-s t} \mathrm{~d} t, \quad \bar{v}=\int_{0}^{\infty} v \mathrm{e}^{-s t} \mathrm{~d} t
$$

The boundary conditions (15) are transformed to

$$
\bar{u}=0, \bar{v}=0 \text { at } y= \pm 1
$$

Solving equations (17) and (18) subject to the boundary conditions (19) we have

$$
\frac{\mathrm{d}^{2} \bar{u}}{\mathrm{~d} y^{2}}-\alpha^{2} \bar{u}=\frac{-C}{(1-\lambda S) S}
$$

where

$$
\alpha^{2}=\frac{S(1+\sigma S)+l s}{(1-\lambda S)(1+\sigma S)}
$$

Finally

$$
\bar{u}=\frac{C}{\alpha^{2} S(1-\lambda S)}\left\{1-\frac{\cosh \alpha y}{\cosh \alpha}\right\}
$$




$$
\bar{v}=\frac{C}{\alpha^{2} S(1-\lambda S)(1+\sigma S)}\left\{1-\frac{\cosh \alpha y}{\cosh \alpha}\right\}
$$

Applying Laplace Inversion formula

$$
u=\frac{1}{2 \pi i} \int_{\delta-i \infty}^{\delta+i \infty} \bar{u} \mathrm{e}^{S t} \mathrm{~d} t
$$

Here $\delta$ is greatest then the real part of all the Singularities of $\bar{u}$

$$
u=\frac{1}{2 \pi i} \int_{\delta-i \infty}^{\delta+i \infty} \frac{C}{\alpha^{2} S(1-\lambda S)}\left\{1-\frac{\cosh \alpha y}{\cosh \alpha}\right\} \mathrm{e}^{S t} \mathrm{~d} t
$$

Taking Inversion Laplace Transform and with the help of calculus of residues the above equations (22) and (23) yields.

$$
\begin{aligned}
\frac{2 u}{C} & =\frac{2}{Q^{2}}\left\{1-\frac{\cosh Q y}{\cosh Q}\right\} \\
& +\frac{8}{\pi} \sum_{r=1}^{\infty} \frac{(-1)^{r} \cos \left(\frac{2 r+1}{2}\right) \pi y\left(1-\sigma S_{1}\right)^{2}\left(1+\lambda S_{1}\right) \mathrm{e}^{-S_{1} t}}{(2 r+1) A_{33}^{\prime}} \\
& +\frac{8}{\pi} \sum_{r=1}^{\infty} \frac{(-1)^{r} \cos \left(\frac{2 r+1}{2}\right) \pi y\left(1-\sigma S_{2}\right)^{2}\left(1+\lambda S_{2}\right) \mathrm{e}^{-S_{2} t}}{(2 r+1) A_{33}^{\prime \prime}}
\end{aligned}
$$

and

$$
\begin{aligned}
\frac{2 v}{C} & =\frac{2}{Q^{2}}\left\{1-\frac{\cosh Q y}{\cosh Q}\right\} \\
& +\frac{8}{\pi} \sum_{r=1}^{\infty} \frac{(-1)^{r} \cos \left(\frac{2 r+1}{2}\right) \pi y\left(1-\sigma S_{1}\right)\left(1+\lambda S_{1}\right) \mathrm{e}^{-S_{1} t}}{(2 r+1) A_{33}^{\prime}} \\
& +\frac{8}{\pi} \sum_{r=1}^{\infty} \frac{(-1)^{r} \cos \left(\frac{2 r+1}{2}\right) \pi y\left(1-\sigma S_{2}\right)\left(1+\lambda S_{2}\right) \mathrm{e}^{-S_{2} t}}{(2 r+1) A_{33}^{\prime \prime}}
\end{aligned}
$$

where

$$
\begin{gathered}
Q=\alpha^{2} \text { at } S \rightarrow 0 \quad A_{33}^{\prime}=\frac{1+l-2 S_{1} \sigma+\sigma^{2} S_{1}^{2}+\lambda S_{1}^{2} \sigma l}{\left(1-S_{1} \sigma+\lambda S_{1}-\lambda S_{1}^{2} \sigma\right)} \\
A_{33}^{\prime \prime}=\frac{1+l-2 S_{2} \sigma+\sigma^{2} S_{2}^{2}+\lambda S_{2}^{2} \sigma l}{\left(1-S_{2} \sigma+\lambda S_{2}-\lambda S_{2}^{2} \sigma\right)} \\
S_{1}=\frac{-X_{1}+X}{2 \sigma\left(1-\pi^{2} \lambda\left(\frac{2 r+1}{2}\right)^{2}\right)}, S_{2}=\frac{-X_{1}-X}{2 \sigma\left(1-\pi^{2} \lambda\left(\frac{2 r+1}{2}\right)^{2}\right)}
\end{gathered}
$$

where

$$
\begin{gathered}
X_{1}=1+l+\pi^{2} \sigma\left(\frac{2 r+1}{2}\right)^{2}-\pi^{2} \lambda\left(\frac{2 r+1}{2}\right)^{2} \\
X=\sqrt{X_{1}^{2}-4 \sigma\left[1-\pi^{2} \lambda\left(\frac{2 r+1}{2}\right)^{2}\right] \times \pi^{2}\left(\frac{2 r+1}{2}\right)^{2}}
\end{gathered}
$$

\section{Results and Discussion}

The unsteady flow of a dusty visco-elastic fluid through a channel is studied. From Figures $\mathbf{1}$ and $\mathbf{2}$ it shows that the velocity profiles for the liquid and the dust particles decreases at different values of time $t$ increases. As the visco-elastic parameter $\lambda$ increases the velocity of the liquid and the dust particles deceases as shown in Figure 3 and 4 . When relaxation time parameter $\sigma$ increases the velocity of the liquid and dust particles decreases as shown in Figure $\mathbf{5}$ and $\mathbf{6}$. From Figure $\mathbf{7}$ and $\mathbf{8}$ it can be observed that as mass concentration increases the velocity of the liquid and the dust particles deceases. In case when gravity or inclination angle $\theta \rightarrow 0$ and viscoelastic parameter $\lambda \rightarrow 0$ and adding the magnetic field term then the present model becomes that of Singh and Ram.

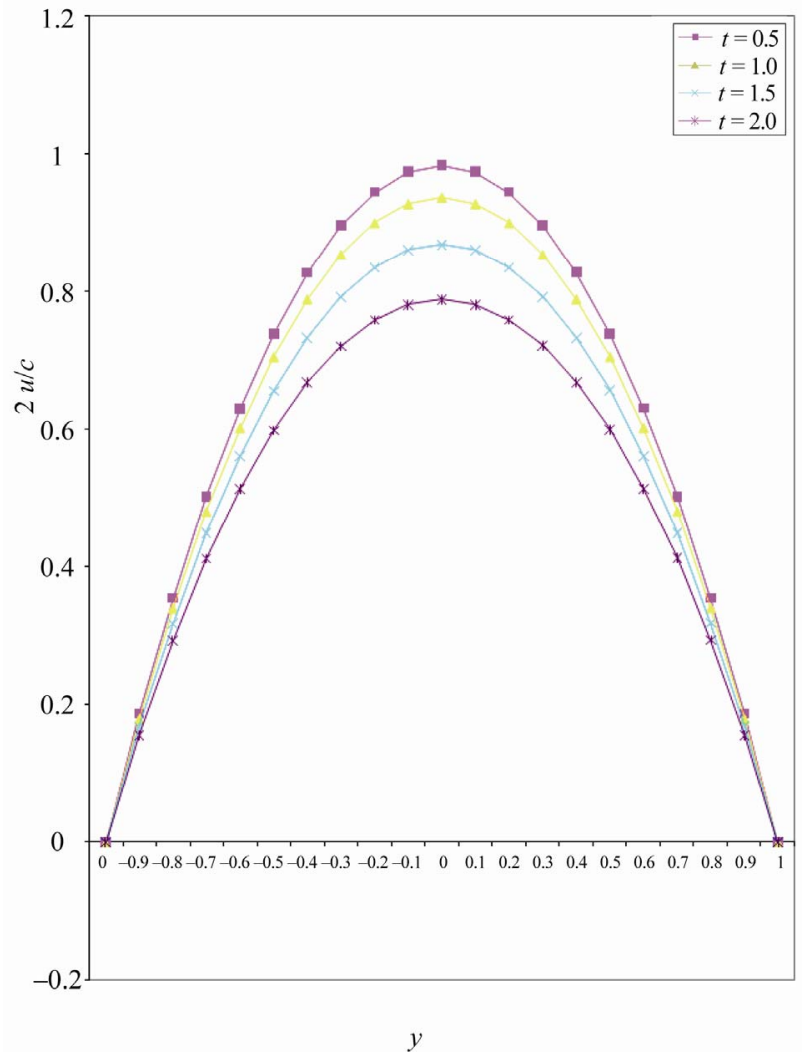

Figure 1. Show the variation of velocity profile of liquid for different value of time at fixed $(\sigma=0.8, \lambda=0.5, I=0.5)$. 


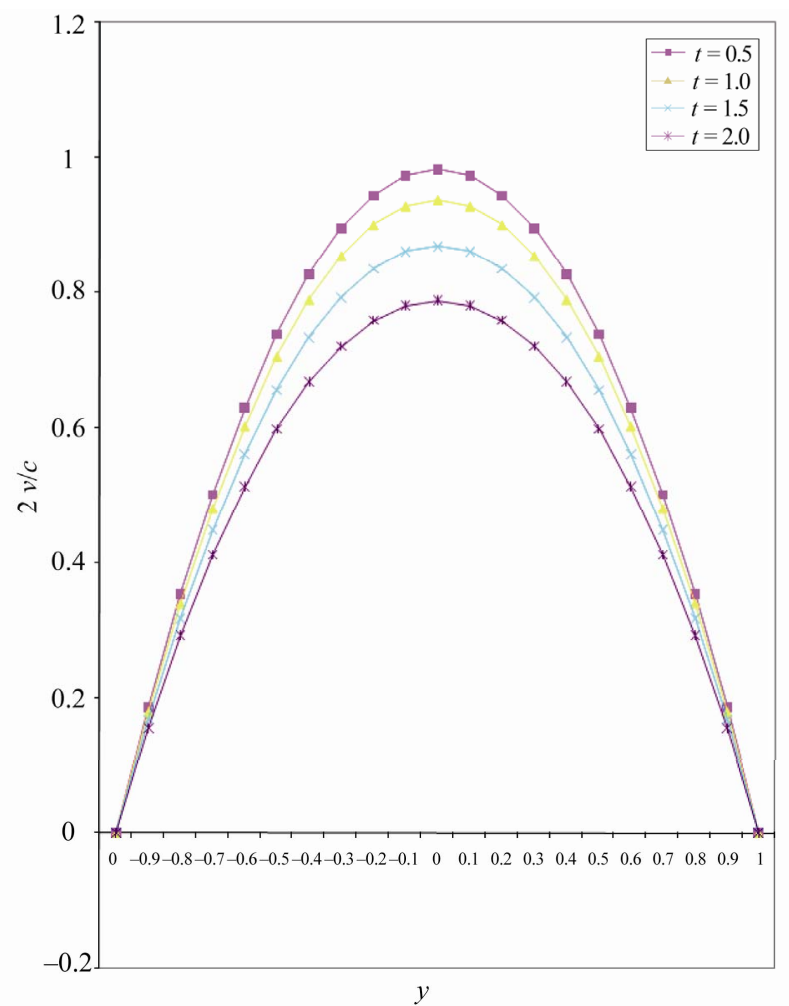

Figure 2. Show the variation of velocity profile of dust for different values of time at fixed $(\sigma=0.8, \lambda=0.5, I=0.5)$.

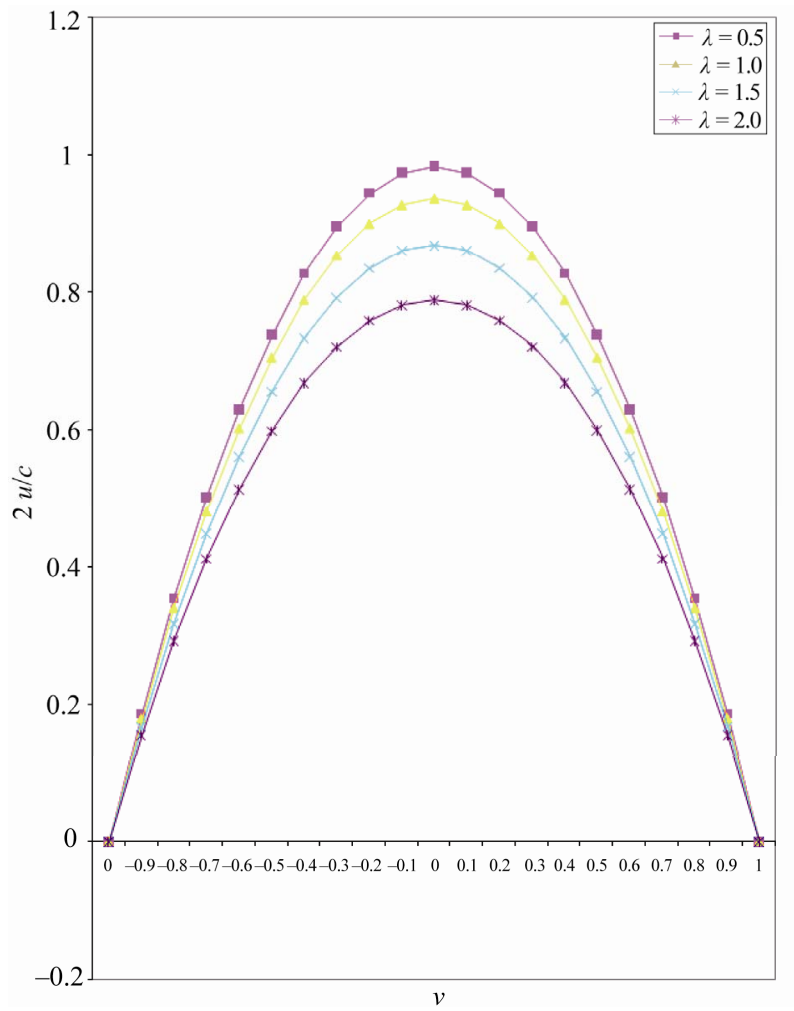

Figure 3. Show the variation of velocity profile of liquid for different values of time at fixed $(\sigma=0.8, \lambda=0.5, I=0.5)$.

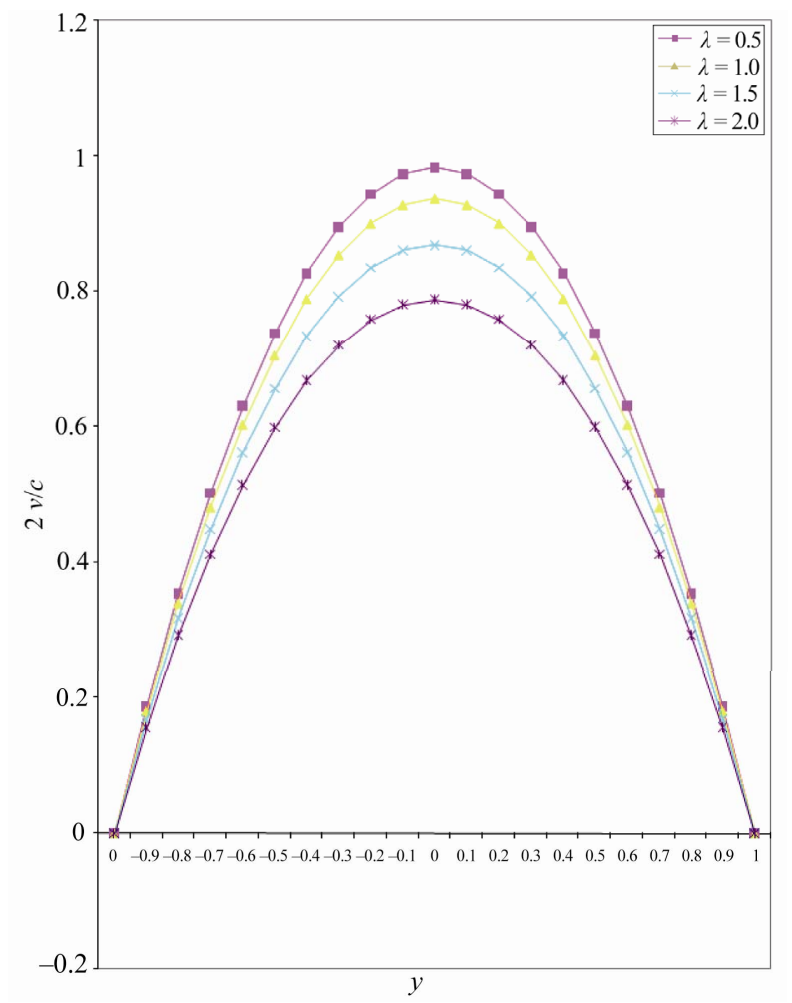

Figure 4. Show the variation of velocity profile of dust for different values of time at fixed $(\sigma=0.8, \lambda=0.5, I=0.5)$.

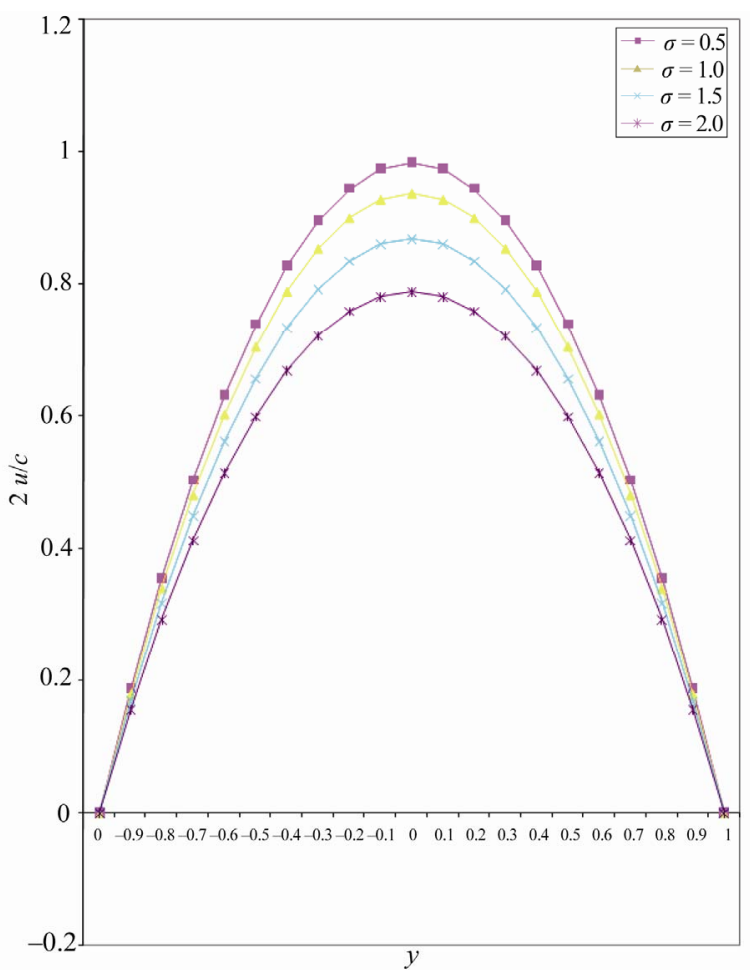

Figure 5. Show the variation of velocity profile of liquid or different values of relaxation and at fixed $(\lambda=0.8, t=0.5, I$ $=0.5$ ). 


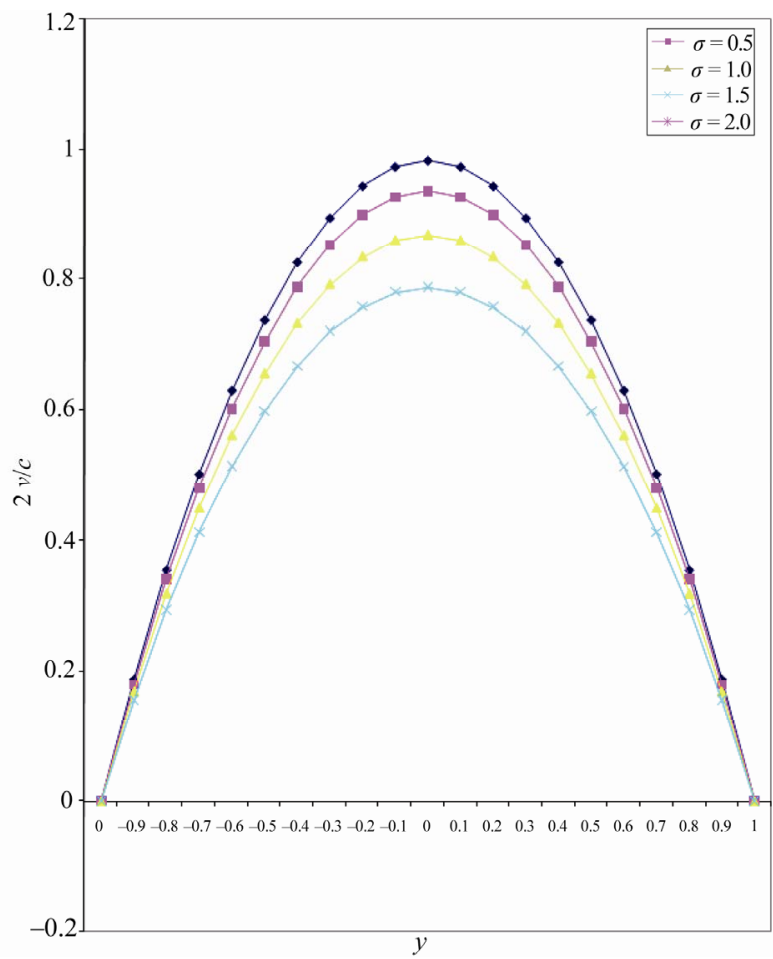

Figure 6. Show the variation of velocity profile of dust or different values of relaxation and at fixed $(\lambda=0.8, t=0.5, I$ $=0.5$ ).

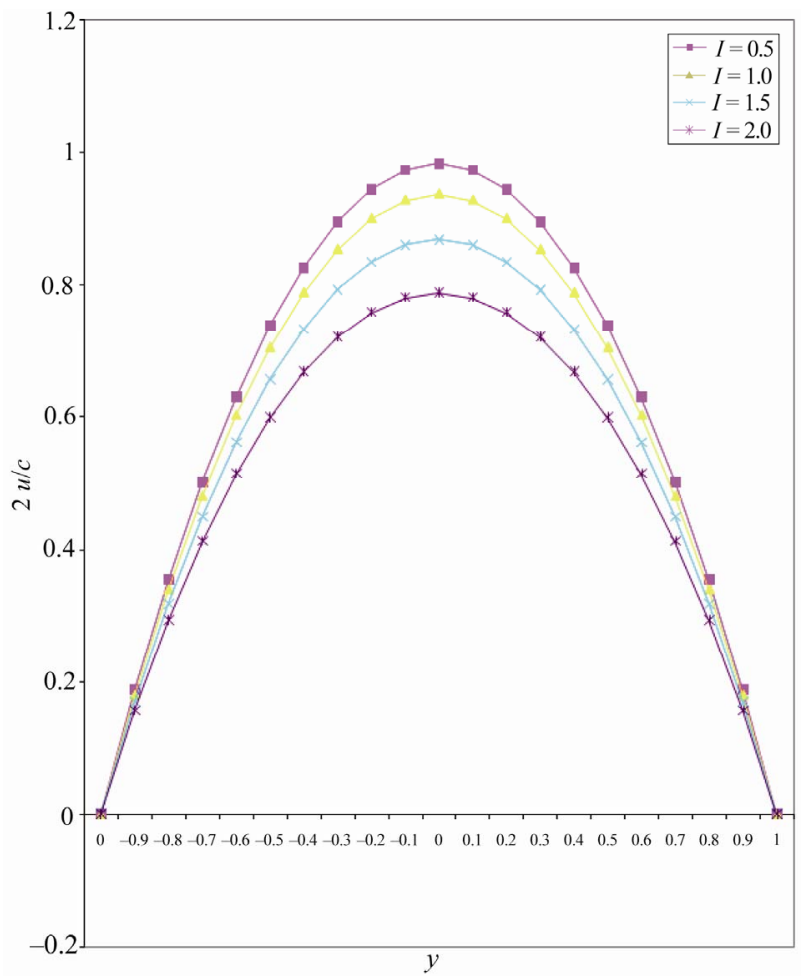

Figure 7. Show the variation of velocity profile of liquid for different values of $I$ mass concentration and at fixed $(\sigma=$ $0.8, t=0.5, \lambda=0.5$ ).

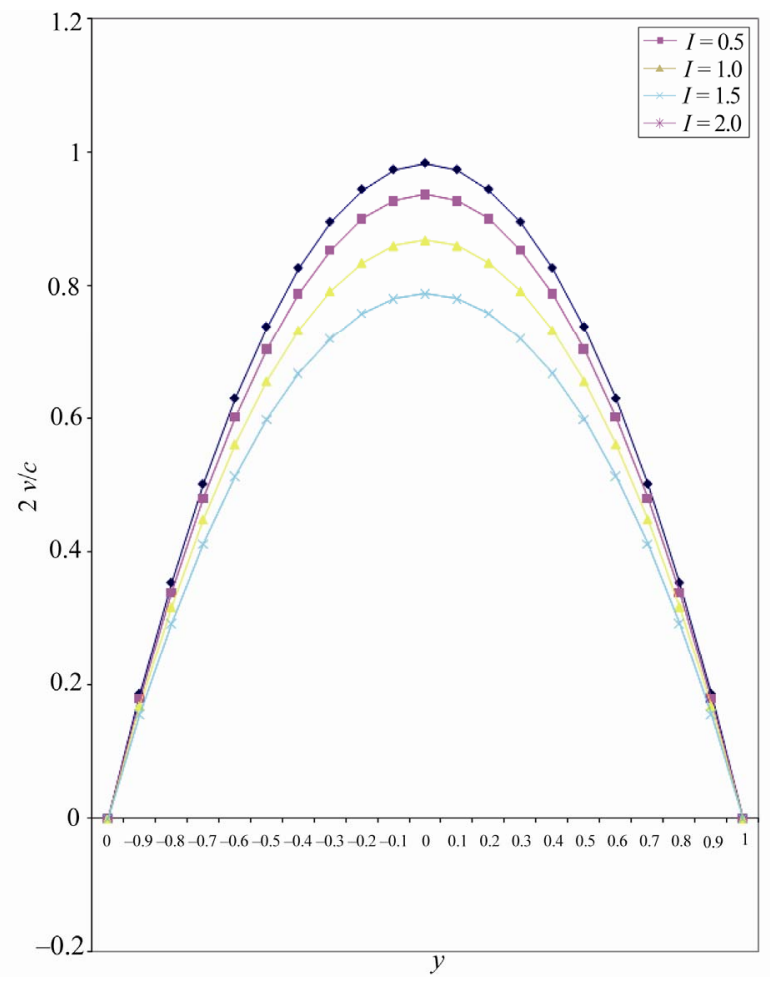

Figure 8. Show the variation of velocity profile of dust for different values of $I$ mass concentration and at fixed $(\sigma=$ $0.8, t=0.5, \lambda=0.5$ ).

\section{References}

[1] D. H. Michael, "Kelvin-Helmholtz Instability of A Dusty Gas,” Proceeding Cambridge Philosophical Sciety, Vol. 61, No. 2, 1965, pp. 569-571. doi:10.1017/S030500410000414X

[2] D. H. Michael and D. A. Millar, "Plane Parallel Flow of a Dusty Gas,” Mathematika, Vol. 13, No. 1, 1966, pp. 97109. doi:10.1112/S0025579300004289

[3] P. G. Saffman, "On stability of a Laminar Flow of a Dusty Gas,” Journal of Fluid Mechanics, Vol. 13, No. 1, 1962, pp. 120-128. doi:10.1017/S0022112062000555

[4] C. B. Singh and P. C Ram, "Unsteady Flow of an Electrically Conducting Dusty Viscous Liquid Through a Channel," Indian Journal of Pure and Applied Mathematics, Vol. 8, No. 9, 1977, pp. 1022-1028.

[5] G. C. Sharma, "Unsteady Flow of an Electrically Conducting Dusty Viscous Liquid between Two Parallel Plates," International Journal of Pure \& Applied Mathematical, Vol. 18, No. 12, 1987, p. 1131.

[6] K. K. Singh, "Unsteady Flow of Conducting Dusty Viscous Liquid in an Annulas," Acta Ciecia Indica, Vol. 3, No. 3, 1977, p. 264.

[7] O. P. Varshney, "Flow of a Dusty Rivlin-Ericksen Fluid through a Channel. Ph.D Thesis,” Agra University, Agra, 1983. 
[8] M. L. Sharma, "MHD Flow of a Conducting Dusty Viscous Liquid through a Long Elliptic Duct with Pressure
Gradient as Function of a Time,” Ph.D Thesis Agra University, Agra, 1980. 